\begin{tabular}{|c|c|c|}
\hline \hline & $\begin{array}{c}\text { International Journal of Current Research in } \\
\text { Biosciences and Plant Biology }\end{array}$ \\
\hline EXCELLENT \\
PUBLISHERS
\end{tabular}

\title{
Study on the Seed Germination Characteristics of Phoebe hunanensis
}

\author{
Chengshi Huang, Bing Sun, Ming Tu and Die Hu*
}

Germplasm Resources Evaluation and Innovation Center of Phoebe Yangtze University, Jingzhou - 434025, Hubei, China

*Corresponding author.

\section{Abstract}

The seed dormancy of phoebe is very long and the reproductive rate is low. In order to study the seed germination characteristics to relieve the dormancy state and improve the germination rate, the seed germination experiment was carried out with Phoebe hunanensis as the material. Research indicated that: The best storage method of Phoebe hunanensis seed was the treatment of wet sand storage; The germination broke about a striking effect when the seeds were soaked in warm water at $35^{\circ} \mathrm{C}$ for $24 \mathrm{hrs}$; Low concentration of gibberellin $\left(\mathrm{GA}_{3}\right)$ solution and naphthalene acetic acid (NAA) solution had little effect on seed germination of Phoebe hunanensis, but the high concentration of gibberellin and naphthalene acetic acid solution were inhibited; the seed of Phoebe hunanensis was germinated earlier and the germination rate was higher when they irradiated with UV-B light than ordinary light, and it was extreme low in dark condition.

\section{Article Info}

Accepted: 30 August 2017

Available Online: 06 September 2017

\section{Keywords}

Germination rate

Phoebe hunanensis

Seed dormancy

\section{Introduction}

Phoebe hunanensis is a kind of small evergreen tree or shrub in Phoebe, Lauraceae, and the heights usually are 3-8 meters. They are mainly distributed in Hunan province, Hubei province, Jiangxi province, Shanxi province and Gansu province in China (Wu et al., 2008). It is suitable for them to grow in a fertile and humid environment, and they are mainly distributed in the valley or water in the broad-leaved forest at an altitude of 500 1000m. The wood of Phoebe is solid, durable and not easy to crack. It is very suitable for making furniture (Wei, 1980). P. hunanensis, as an important species of Phoebe, maintains a lot of fine characters. It has a great potential for exploitation and utilization. At present, there are few studies on $P$. hunanensis all around the world and the research on seed germination characteristics has not been reported previously. Zhang et al. (2016) had only made a preliminary study on its seed morphological characteristics compared with other phoebe plants. In order to explore the characteristics of $P$. hunanensis' seed germination, the experiment was composed of five parts. The treatments are different seed storage methods, different seed soaking times, different concentrations of $\mathrm{GA}_{3}$ and NAA solution, different light conditions irradiation. It is of practical significance to seed breeding of phoebe, and it is also beneficial to popularize and utilize for this tree species.

\section{Materials and methods}

\section{Experiment material}

The seeds of the experiment were collected from the Jiugongshan National Nature Reserve in Xianning City, Hubei Province, China. Then put the seeds soaked in the 
water and removed the peel. After that, choosing the particles plump seeds that had not been rotten placed in the ventilated shade until they were dry, and then making different treatments for the germination test. This experiment began on November 24, 2016, and ended on April 14, 2017.

\section{Experiment methods}

In the first place, making the seeds sterilized with $5 \mathrm{~g} / \mathrm{L}$ potassium permanganate solution after 30 minutes, and then rinsed with water. The seeds spread evenly until they were naturally dry. The seeds were treated in three different ways, namely, natural room temperature dry storage, low temperature $\left(4^{\circ} \mathrm{C}\right)$ cold storage and wet sand storage. In the second place, choosing the best way of stored seeds above, and conducting an experimental study on seed germination of different concentrations of $\mathrm{GA}_{3}$ and NAA solution, different soaking times and different light conditions.
Matting two sheets of wet filter paper onto clean culture dishes $(\mathrm{d}=9 \mathrm{~cm})$ and placing the seeds evenly on culture dishes with tweezers, after that, covering the lid to preserve moisture. The culture dishes were put in the light constant temperature culture room. The temperature of the room is constant $25^{\circ} \mathrm{C}$, and the humidity of the room is $70 \%$. Observing the seeds every day and adding moderate water on filter paper for moisturizing. The filter paper was replaced and the musty seeds were cleaned or removed timely. This experiment took it as germination when the radical had broken through seed coat, and the seed germination was observed and recorded every day. It wound be considered the end of germination if the seed did not germinate for five consecutive days ( $\mathrm{Li}$ et al., 2012). It was used 150 grains of seeds for each treatment, repeated three times and 50 grains of seeds each repetition. The test results were taken the average of three times, and the germination rate was analyzed by variance. The germination rate and germination potential were calculated according to the following formula (ISTA, 1996).

Germination rate $=($ number of seeds forming normal seedlings $/$ total number of tested seeds $) \times 100 \%$

Germination potential $=($ number of germinated seeds at the onset of germination to peak number of buds/total number of tested seeds $) \times 100 \%$

\section{Study on seed physical properties}

The study of the seed physical characteristics of $P$. hunanensis was carried out according to the Chinese standard. Observing and describing the typical morphology of the seeds, and then, determining the thousand seed weight (TKW). According to the rules of Chinese forest seed inspection, the seeds were randomly selected from mature and healthy seeds, repeated three times and 100 grains of seeds each time, and then were weighted with electronic scales, accurate to $0.001 \mathrm{~g}$, then taking the average and calculating the TKW.

\section{Different storage methods}

The seeds were evenly stored in three different ways: natural room temperature dry storage, low temperature $\left(4^{\circ} \mathrm{C}\right)$ cold storage and wet sand storage respectively. Natural room temperature dry storage means placing the sterilized seeds into a closed brown glass bottle for dry storage. Low temperature cold storage means placing the seeds in a transparent glass bottle, and then put it into the $4^{\circ} \mathrm{C}$ constant temperature refrigerator for cold storage. Wet sand storage means putting the seeds into the wet sand treated with carbendazim, and the humidity was proper that it was not loose and leaking when handing the wet sand, by the way of adding one layer of wet sand onto one layer of seeds, and then put them into the flower pot. The three treatments had a same storage time of 90 days. After the storage, placing the seeds, which were divided into three groups, into the culture dishes and putting them in the light constant temperature culture room. Then observing and noting their germination state at the same time every day.

\section{Different seed soaking time}

The seeds were immersed in water at $35^{\circ} \mathrm{C}$, and then soaking $6 \mathrm{hrs}, 12 \mathrm{hrs}, 24 \mathrm{hrs}, 48 \mathrm{hrs}$ and no soaking treatment of five treatments in total. After soaking time, removing and putting the seeds into the culture dishes. Then we translated them into the light constant temperature culture room. The illumination was set a time for $16 \mathrm{hrs}$ each day.

\section{Different plant hormone treatment}

The seeds were immersed respectively in $\mathrm{GA}_{3}$ and NAA solution with different mass fractions of $100 \mathrm{mg} / \mathrm{L}, 200$ $\mathrm{mg} / \mathrm{L}, 300 \mathrm{mg} / \mathrm{L}, 400 \mathrm{mg} / \mathrm{L}$ and $500 \mathrm{mg} / \mathrm{L}$ for $24 \mathrm{hrs}$. We regarded it as blank control group (CK) that immersed in distilled water. The processed seeds were divided into different groups and placed in the light constant temperature culture room. The illumination was set a time for $16 \mathrm{hrs}$ each day. 


\section{Different light conditions}

In the light constant temperature culture room, setting the seeds irradiated under three different light conditions, respectively, continued darkness, exposed to the ordinary light for $16 \mathrm{hrs}$ each day, exposed to UV-B light for $16 \mathrm{hrs}$ each day, then observing and noting their germination state at the same time every day.

\section{Results and analysis}

\section{Seed physical characteristics}

The mature $P$. hunanensis seeds were ovoid, seed coat was dark brown, and seed ridge was not obvious. The length of the seeds was $10.2 \sim 11.7 \mathrm{~mm}$ and the width of the seeds was $5.6 \sim 7.2 \mathrm{~mm}$. The thousand seed weight was $287.4 \mathrm{~g}$, belonged to medium-sized seed.

\section{Effects of different storage methods on seed germination}

It can be seen from Table 1, the germination rate and germination potential arranged from high to low treatment followed by wet sand storage > low temperature cold storage $>$ natural room temperature dry storage. Different storage methods on $P$. hunanensis demonstrated the significant effect of seed germination and germination potential, wet sand storage and low temperature cold storage can significantly increase the rate of seed germination compared with natural room temperature dry storage, and germination rate reached respectively $89.4 \%, 84.2 \%$. Analysis of variance (the alpha was at a level at 0.05 ) showed that there was no significant difference on seed germination between wet sand storage and low temperature cold storage, but it was greatly different with natural room temperature dry storage. The germination potential of the three different storage methods had significant difference.

Table 1. Effects of different storage methods on seed germination of P. hunanensis.

\begin{tabular}{lll}
\hline Storage method & Germination rate (\%) & Germination potential (\%) \\
\hline Natural room temperature dry storage & $46.6 \mathrm{~b}$ & $24.0 \mathrm{c}$ \\
Low temperature cold storage & $84.2 \mathrm{a}$ & $50.6 \mathrm{~b}$ \\
Wet sand storage & $89.4 \mathrm{a}$ & $61.5 \mathrm{a}$ \\
\hline
\end{tabular}

The same letters were shown there was no significant difference in analysis of variance (the alpha was at a level at 0.05). But the different letters were shown significant difference.

\section{Effect of different soaking time on seed germination}

The germination rate of $P$. hunanensis was different after different seed soaking time. As can be seen from Fig. 1, when the soaking time respectively were $6 \mathrm{hrs}, 12 \mathrm{hrs}, 24$ hrs, the seed germination rate gradually increased. But in the soaking time for $48 \mathrm{hrs}$, the germination rate had a decreasing tendency. The optimum seed soaking time was $24 \mathrm{hrs}$ and the germination rate was $89 \%$, which was higher than that of no soaking. This showed that soaking seeds can remove the seed dormancy and increase germination rate to a certain extent.

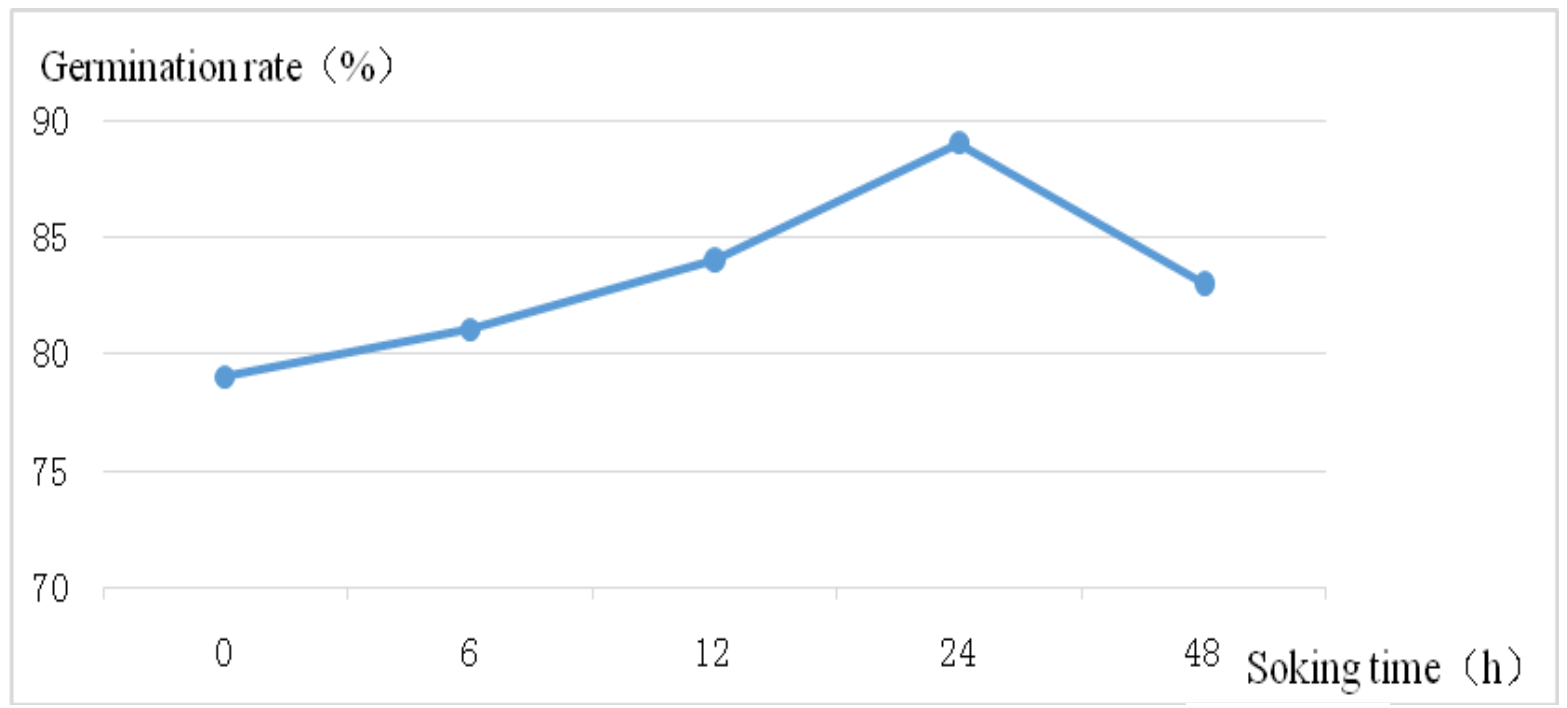

Fig. 1: Effect of different seed soaking time on seed germination rate of $P$. hunanensis. 


\section{Effects of different plant hormones on seed germination}

Fig. 2 shows the effect of different concentrations of $\mathrm{GA}_{3}$ and NAA solution on the seed germination of $P$. hunanensis. It could be seen from the figure that low concentration of $\mathrm{GA}_{3}$ and NAA solution had little effect on seed germination, but the germination rate showed a decreasing trend when both of the concentration exceeded $300 \mathrm{mg} / \mathrm{L}$, which indicating that high concentrations of $\mathrm{GA}_{3}$ and NAA solution had an inhibition effect on the seed germination. In the same concentration gradient, the seed germination rate of $\mathrm{GA}_{3}$ solution was higher than that of NAA solution, which indicating that $\mathrm{GA}_{3}$ had better effect on seed germination of $P$. hunanensis than NAA.

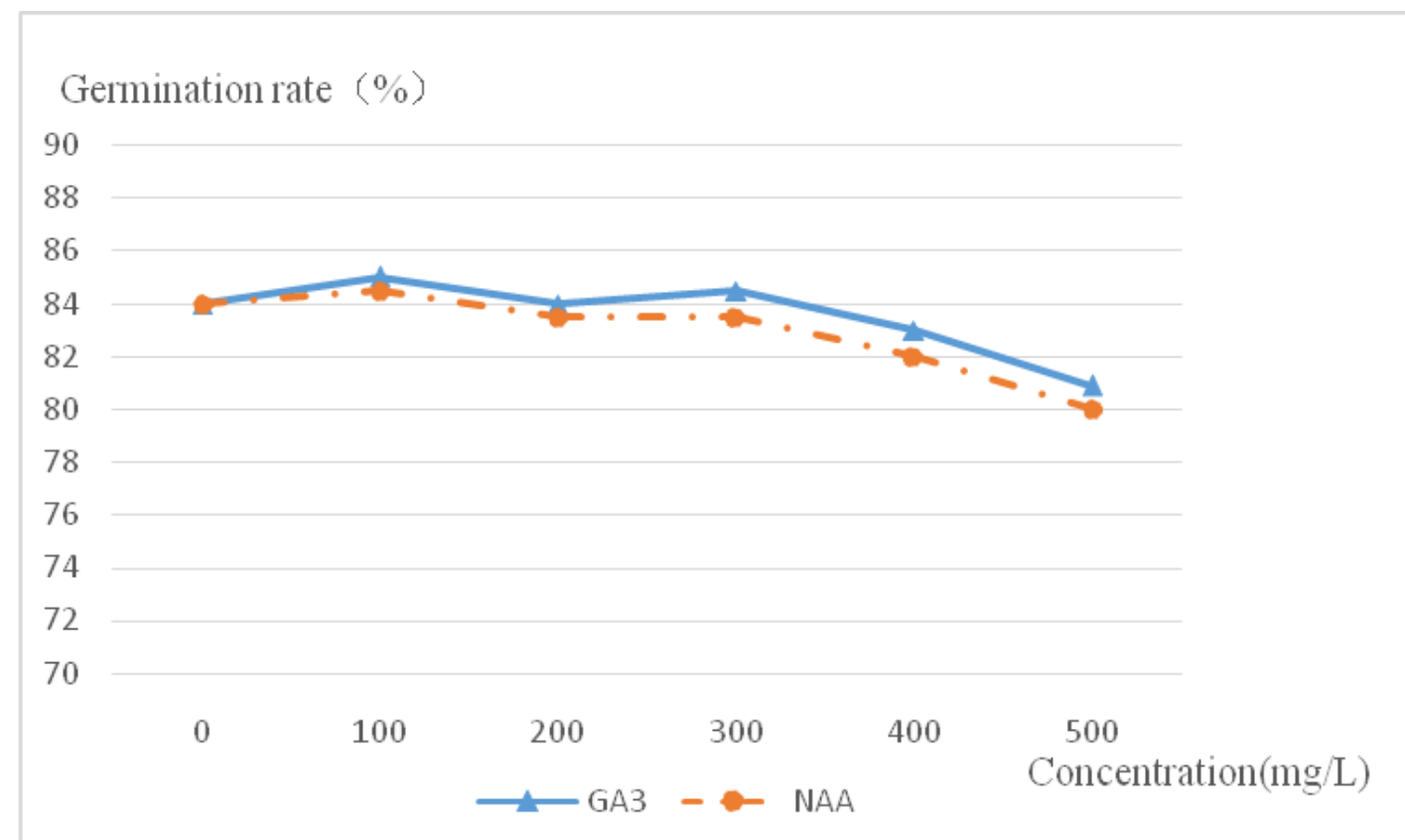

Fig. 2: Effect of different concentrations of $\mathrm{GA}_{3}$ and NAA solution on seed germination of $P$. hunanensis.

\section{Effect of different light conditions on seed germination}

Table 2 shows that the best result of $P$. hunanensis seed germination was under the condition of UV-B light irradiation, the germination rate and germination potential are respectively $91.6 \%$ and $65.8 \%$, followed by ordinary light irradiation and the lowest germination rate was under dark condition. The results of variance analysis (the alpha was at a level at 0.05) showed that the seed germination rate and germination potential of $P$. hunanensis were significantly different when they were irradiated under the condition of UV-B light, ordinary light and dark condition.

Table 2. Effects of different light conditions on seed germination of P. hunanensis.

\begin{tabular}{lll}
\hline Light conditions & Germination rate (\%) & Germination potential (\%) \\
\hline Duck condition & $56.8 \mathrm{c}$ & $29.2 \mathrm{c}$ \\
Ordinary light irradiation & $82.4 \mathrm{~b}$ & $53.2 \mathrm{~b}$ \\
UV-B light irradiation & $91.6 \mathrm{a}$ & $65.8 \mathrm{a}$ \\
\hline The same letters were shown there was no significant difference in analysis of variance (the alpha was at a level at 0.05$).$ But \\
the different letters were shown significant difference.
\end{tabular}

\section{Summary and discussion}

The When a viable seed was in a suitable germination condition, but they did not germinate normally, it is said that the seed was in a dormant state (Yan, 1995). Just like many seeds of Phoebe, the seed of $P$. hunanensis also has dormancy characteristics, however, breaking seed dormancy is an important way to improve seed germination rate. The method of seed dormancy was discussed by different seed storage methods, different 
seed soaking times, different plant hormones and different light conditions. The results are as follows:

(1) Different storage methods had obvious difference in germination rate and germination potential of $P$. hunanensis, and the best effect was found in wet sand storage for 90 days. The results were the same as those of Feng et al. (2005) on seed germination characteristics of Machilus pauhoi. The seed coat would soften after the wet sand storage, and its permeability and breathability were enhanced, which was conducive to breaking the seed dormancy to germination. However, the seeds after wet sand storage were easier to become mildew and rot than the seeds after low temperature cold storage. In one word, wet sand storage and low temperature cold storage are good choices for long-term storage of seeds.

(2) Seed germination begins with water swelling $(\mathrm{Xu}$ et al., 2014). From the results of this experiment, different seed soaking time had an effect on seed germination rate of $P$. hunanensis. The seed germination rate increased with the increase of soaking time, but there was a tendency to decrease after the first increase. The best seed soaking time was $24 \mathrm{hrs}$, and the germination rate was up to $89 \%$. Xu et al. (2017) studied the seed germination of $P$. zhennan; they set three treatments, respectively, no soaking, soaking $12 \mathrm{hrs}$ and soaking 24 hrs. Their result showed that the best soaking time is also $24 \mathrm{hrs}$. Indicating that soaking seeds plays the role of spouting, and soaking time can affect the seed germination rate of Phoebe to a large extent.

(3) The two plant hormones of $\mathrm{GA}_{3}$ (Yu et al., 2003) and NAA (Li, 2010) were widely used in forestry production, and both of them have a certain regulating effect of forest seed germination. However, low concentration of $\mathrm{GA}_{3}$ and NAA solution neither had little effect on seed germination of $P$. hunanensis while high concentration of $\mathrm{GA}_{3}$ and NAA solution had a tendency to inhibit the seed germination. The same concentration of NAA solution was more sensitive than $\mathrm{GA}_{3}$ solution to germination inhibition, which indicated that $\mathrm{GA}_{3}$ was easier to relive the seed dormancy of $P$. hunanensis than NAA.

With the climate changing, the UV-B radiation on the earth's surface was enhanced, the growth of animals and plants caused a wide range of effects (Zhang et al., 2009), light is also a major factor affecting plant seed germination (Fu et al., 2009), through different lighting conditions on the test of $P$. hunanensis. Seed germination showed that light conditions had a significant effect on the seed germination. Under the condition of UV-B light irradiation, the seed germination rate and germination potential were up to $91.6 \%$ and $65.8 \%$, which were much higher than those in common light and dark conditions, and the seed germination was also the earliest. This shows that UV-B radiation can break the seed dormancy of $P$. hunanensis and improve its germination rate. However, at the later stage of seed germination, it was found that the seeds under UV-B light irradiation were more prone to become mildew and rot, indicating that UV-B radiation had a certain degree of damage to the seeds for a long time and we should properly control the time of UV-B light irradiation.

\section{Conflict of interest statement}

Authors declare that they have no conflict of interest.

\section{Acknowledgement}

This work was supported by the national second survey for key protected wild plant resources (special investigation for Phoebe and Machilus in Hubei Province), the Science and Technology Support Program in Hubei Province of P. R. China (2013BBB24).

\section{References}

Feng, J. M., He, P. G., Luo, W. J., Chen, Y. T., Chen, Y. L., Ma, J. Q., 2005. Preliminary report on flowering and fruiting habits and seed storage of Machilus pauhoi. J. Zhejiang For. Sci. Technol. 3, 23-25.

Fu, T. T., Cheng, H. Y., Song, S. Q., 2009. Research progress of seed dormancy. Chin. Bull. Bot. 5, 629641.

ISTA (International Seed Testing Association), 1996. International Code for Seed Inspection. Agric. Univ. Press, Beijing.

Li, C. L., 2010. Application of naphthylacetic acid in forestry production. Mod. Agric. Sci. Technol. 23, 28-29.

Li, Z., Wang, S. J., Liu, C. L., Lin, X. Z., 2012. Study on seed germination characteristics of Phoebe sheareri and Phoebe chekiangensis. Northern. Hort. 7, 58-60.

Wei, F. N., 1980. Classification of Phoebe Nees plants in China. Guihaia. 1, 46-59.

Wu, Z. Y., Raven, P. H., Hong, D. Y., 2008. Lauraceae. 
In: Flora of China. Vol. 7. Science Press, Beijing and Missouri Botanical Garden Press, St. Louis. pp.102-254.

Xu, H. H., Li, N., Liu, S. J., Wang, W. Q., Wang, W. P., Zhang, H., Cheng, H. Y., Song, S. Q., 2014. Advances in seed germination and its regulation. Acta Agric. Sci. 7, 1141-1156.

Xu, J., Chen, H. X., He, F., Gao, H. Z., Li, L. Z., 2017. The seed germination test of Phoebe zhennan. Trop. For. 1, 16-18.

Yan, Y. M., 1995. Summary of seed dormancy. Seed. 4,
30-34.

Yu, Z. X., Huang, X. Q., Fang, Z. S., Jiang, C. G., Zhang, X. E., 2003. The research progress of GA on the germination of forest seeds. J. Zhejiang For. Sci. Technol. 1, 73-76.

Zhang, J., Wang, J., Tian, L. P., 2009. Advances in research on plant growth by enhanced UV-B radiation. Chin. Agric. Sci. Bull. 22, 104-108

Zhang, J., Xu, S. Y., Li, G., Wang, Q., Fei, Y J., 2016. Seed characteristics of five species of Phoebe and its significance in classification. Seed. 2, 50-53.

\section{How to cite this article:}

Huang, C., Sun, B., Tu, M., Hu, D., 2017. Study on the seed germination characteristics of Phoebe hunanensis. Int. J.

Curr. Res. Biosci. Plant Biol. 4(9), 15-20. doi: https://doi.org/10.20546/ijcrbp.2017.409.003 